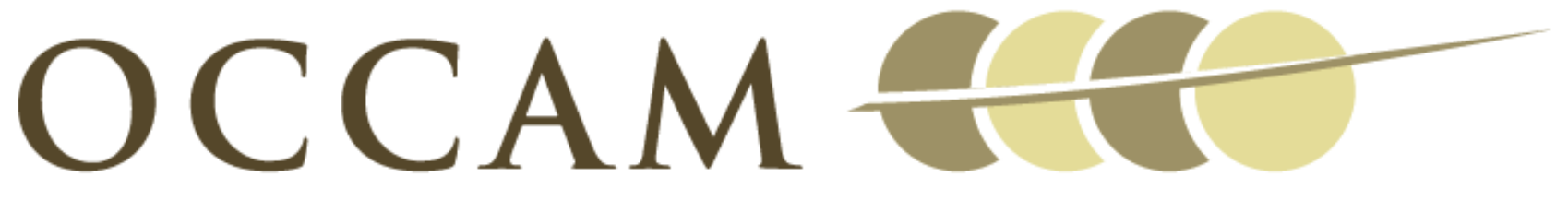

OXFORD CENTRE FOR COLLABORATIVE APPLIED MATHEMATICS

Report Number 12/20

Multiple equilibria in a simple elastocapillary system

by

Michele Taroni and Dominic Vella

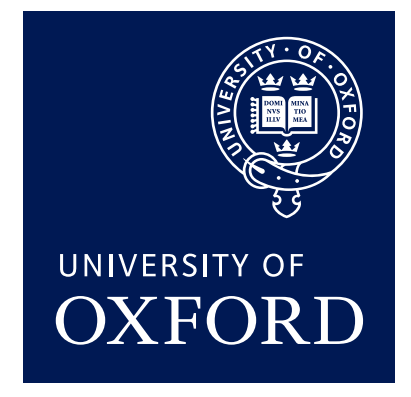

Oxford Centre for Collaborative Applied Mathematics Mathematical Institute 24 - 29 St Giles'

Oxford OX1 3LB

England 



\title{
Multiple equilibria in a simple elastocapillary system
}

\author{
MICHELE TARONI AND DOMINIC VELLA ${ }^{1,2} \dagger$ \\ ${ }^{1}$ Oxford Centre for Collaborative Applied Mathematics, Mathematical Institute, \\ 24-29 St Giles', Oxford, OX1 3LB, UK \\ ${ }^{2}$ Department of Applied Mathematics and Theoretical Physics, University of Cambridge, \\ Wilberforce Rd, Cambridge, CB3 0WA, UK \\ (Received ?; revised ?; accepted ?.)
}

\begin{abstract}
We consider the elastocapillary interaction of a liquid drop placed between two flexible sheets, which are both clamped at one end to a rigid substrate. This is a simple model system relevant to the problem of surface tension-induced collapse of flexible microchannels that has been observed in the manufacture of microelectromechanical systems (MEMS). We determine the conditions under which the flexible sheets remain separated, touch at a point, or stick along a portion of their length. Surprisingly, we show that in many circumstances multiple equilibrium states are possible. We develop a lubricationtype model for the flow of liquid out of equilibrium and thereby investigate the stability of the multiple equilibria. We demonstrate that for given material properties two stable equilibria may exist and show through numerical solutions of the dynamic model that it is the initial state of the system that determines which stable equilibrium is reached by the system; the system does not simply choose the equilibrium state of lowest energy.
\end{abstract}

\section{Key words:}

\section{Introduction}

Recently, there has been great interest in the interaction between elastic and capillary forces, commonly referred to as 'elasto-capillarity'. While this activity has primarily been driven by applications at the microscopic scale (where objects are often sufficiently flexible that surface forces are strong enough to bend them) there are also a number of everyday situations in which elasto-capillary effects can be observed. For example, the bending of paintbrush hairs caused by wetting with a liquid motivated several experiments to investigate the elasto-capillary analogue of capillary rise (Bico et al. 2004; Kim \& Mahadevan 2006). Most often, studies of elasto-capillary phenomena have focussed on understanding the various static configurations that arise. However, more recently attention has shifted to understand the fluid motions that occur in such problems. The dynamics of elasto-capillary rise, in which gravity leads to a finite final meniscus height, were studied by Duprat, Aristoff \& Stone (2011). In the absence of gravity the liquid meniscus continues to move until it reaches the edge of the system; such motion has been studied by van Honschoten et al. (2007) and Aristoff, Duprat \& Stone (2011).

At the microscopic scale, the manufacture of microelectromechanical systems (MEMS) frequently involves the interaction between the surface tension of a solvent and the elasticity of the components of a MEMS device. This can happen either in the fabrication

$\dagger$ Email address for correspondence: dominic.vella@maths.ox.ac.uk 

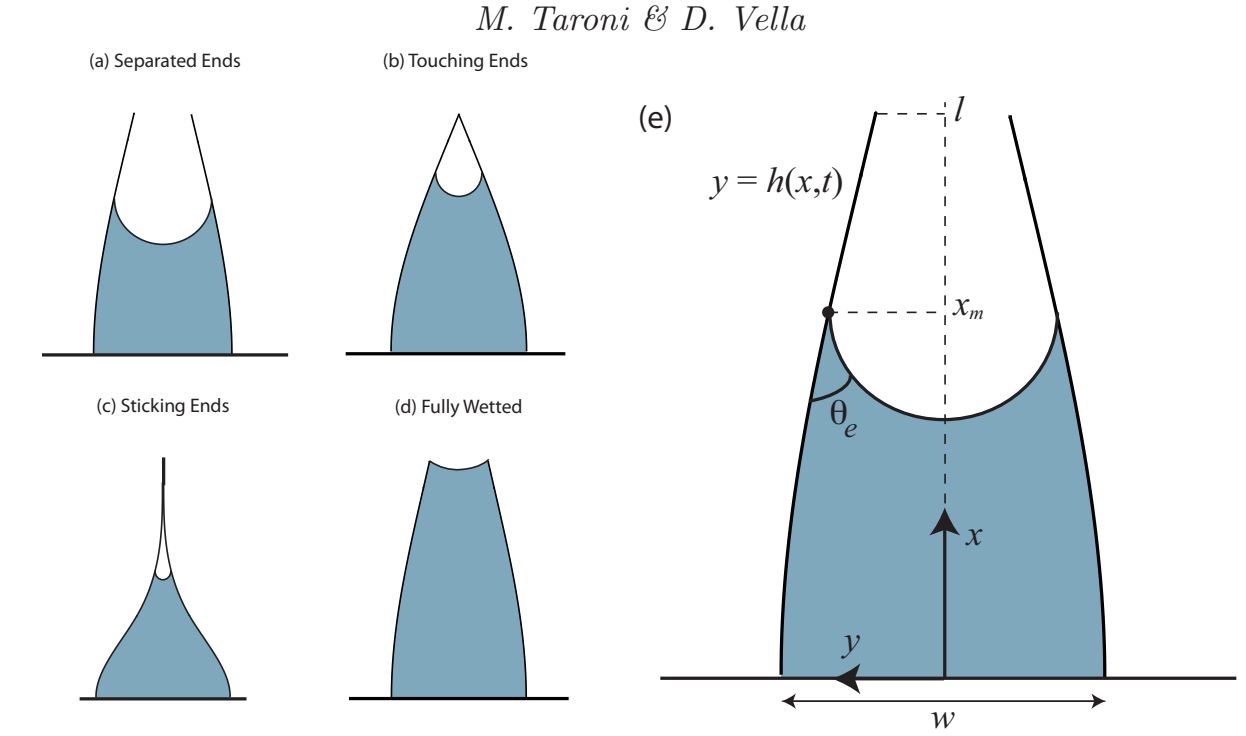

Figure 1. (a)-(d) The four possible regimes for a volume of liquid confined between two elastic micro-pillars and a rigid base. (e) The notation used in the theoretical formulation of the problem. A two-dimensional microchannel of width $w$ and length $\ell$ is filled with a volume $v$ (per unit length) of liquid. The liquid meniscus is located at $x=x_{m}$ and maintains a constant contact angle $\theta_{e}$ with the beam that makes up the wall of the channel.

of a 'mask' using photolithography (Berkowski, Plunkett, Yu \& Moore 2005) or in the stamping of a soft material such as polydimethylsiloxane (PDMS) using such a mask (Xia \& Whitesides 1998). In both cases, patterns are created involving relatively slender beams, which are susceptible to sticking together when a liquid meniscus is introduced since the surface tension of the liquid pulls neighbouring beams together. This can occur when either an etching agent (Mastrangelo \& Hsu 1993a,b; Raccurt et al. 2004) or a rinsing agent (Tanaka et al. 1993; Delamarche et al. 1997; Lee et al. 2002) dries; the effect is similar in either case. It has also been shown that the 'stiction' caused in this way can sometimes be reversed by rinsing with a different liquid (Delamarche et al. 1997).

In this paper we consider two beams that are clamped to a rigid substrate forming a 'micro-channel' of width $w$. A finite volume of liquid is deposited at the base of the channel in such a way that the surface of the liquid lies within the channel. The two beams are thus elastically deformed by the surface tension of the interface, as shown schematically in figure 1. In practical applications, deformation can occur because of capillary condensation as well as the rinsing and drying of components (van Spengen et al. 2002). This may cause a droplet to be stuck near the tip of the beams, a situation that has been studied previously by Kwon, Kim, Puell \& Mahadevan (2008). However, in general there is no equilibrium configuration with the droplet midway along the beam. For simplicity, therefore, we shall consider the droplet to be in contact with the rigid base of the micro-channel, as shown in figure 1. As a further simplification, we shall neglect the effect of evaporation so that the volume of the droplet remains constant. Finally, we neglect the effect of gravity since the micro-channels considered are significantly below the scale at which either hydrostatic pressure within the liquid or the bending of the beams due to their weight become important.

The different configurations that may be adopted by the flexible beams that bound the micro-channel are illustrated in figure 1 . We expect that for very small liquid volumes the beams will be bent but remain separated along their length (as depicted in fig. 1a). 
As the volume of liquid increases we expect that the distance between the two free ends should decrease. Eventually, we might expect the two beams to touch (see figure 1b) or even 'stick' along a finite portion of their length (figure 1c). We note that the model we develop does not include an adhesive force between the two beams. However, this geometrical sticking caused by the macroscopic liquid meniscus allows the beams to come into close enough contact for microscopic van der Waals forces to become important and stick the beams irreversibly once the liquid has been completely removed. It is thus the initial contact between beams that may lead to problems in the operation of MEMS devices. At the largest droplet volumes we expect the beams to be fully wetted by the liquid (figure 1d). In this case the concept of an equilibrium contact angle $\theta_{e}$ is not well defined (Mansfield et al. 1997). In principle, the angle that the meniscus makes to the horizontal could be found as part of the solution, but given that, within the context of our model, there is no mechanism (e.g. gravity) to determine the limits of this configuration, we do not consider this regime here.

In this paper, we investigate the conditions under which each of the three configurations in figure 1a-c are observed. We show that in certain circumstances several different equilibrium configurations exist; to investigate which of these is realized we develop a model of the liquid flow based on lubrication theory. We then perform a stability analysis, which demonstrates that multiple stable equilibria exist. Using full numerical solutions of the dynamic problem, we show that which equilibrium the system reaches then depends on the initial condition for the system. While the existence of multiple equilibria has been reported in related systems previously (see Mastrangelo \& Hsu 1993a; Py et al. 2007; Kwon et al. 2008, for example) ours is, to the best of our knowledge, the first study that makes use of a dynamic model to investigate the stability of these multiple equilibria. In particular, this demonstrates that the system does not necessarily tend to the state of lowest energy.

The plan of the paper is as follows. In $\S 2$ we describe our theoretical formulation of the problem by developing a lubrication model for the motion of liquid trapped within a micro-channel and obtain the equations governing the equilibrium situation as the steady limit of the dynamic problem. We then consider two different values for the equilibrium contact angle $\theta_{e}$, allowing us to isolate two different force contributions arising from surface tension. In $\S 3$ we study the case $\theta_{e}=\pi / 2$, in which case surface tension causes a line force to act at the contact line. In $\S 4$ we study the case $\theta_{e}=0$, where surface tension causes a capillary pressure to act along the wetted length of the beam but no line force. The results of these two cases are qualitatively similar though differ in some of the details. Finally, in $\S 5$ we summarize our results and consider briefly their practical implications.

\section{Theoretical formulation}

\subsection{Beams subject to surface tension forces}

We consider the two dimensional situation shown in figure 1e: a volume per unit length $v$ of liquid is placed between two elastic beams of length $\ell$ clamped a distance $w$ apart. We imagine that the contact line is at a position $x_{m}$ and that the contact angle of the liquid on the beam has an equilibrium value $\theta_{e}$. We assume that the gradient of the beam deflections remain small throughout so that we may use linear beam theory (see, for example, Landau \& Lifschitz 1959) to describe the shape of each beam. Two forces act on each beam as a result of the surface tension $\gamma$ of the liquid: a line force $\gamma \sin \theta_{e}$ acts at the contact line, while a capillary pressure force $\gamma \kappa$ due to the curvature $\kappa$ of the 
interface, acts just beneath the contact line. In equilibrium, the capillary pressure acts all along the wetted length of the beam. We note that the majority of previous analyses have neglected the line force and focussed instead on the force arising from the integrated curvature pressure, although Farshid Chini \& Amirfazli (2010) considered both. Here we consider separately the limits $\theta_{e}=\pi / 2$, in which case only the line force acts on the beam, and $\theta_{e}=0$, in which case only the capillary pressure acts on the beam. These two limits might be expected to span the range of possible behaviours for this system and indeed have qualitatively similar behaviour, as we shall see.

Our starting point is to consider the scaling behaviour for the beam length for which we expect surface tension to cause the beams to interact. For deformations of the beams with small slope, the beam equation (Landau \& Lifschitz 1959) governs the shape of each beam $y=h(x)$ according to

$$
B h^{\prime \prime \prime \prime}(x)=p(x)-\gamma \sin \theta_{e} \delta\left(x-x_{m}\right),
$$

where $B$ is the bending stiffness per unit length and

$$
p(x)= \begin{cases}p_{\text {liquid }}, & 0<x<x_{m} \\ 0, & x_{m}<x<\ell\end{cases}
$$

is the pressure within the liquid (measured relative to the atmospheric pressure). The Dirac delta function, $\delta(x)$, accounts for the line force arising from surface tension $\gamma$. For static configurations the pressure within the liquid, $p_{\text {liquid }}$, is uniform and given by

$$
p_{\text {liquid }}=-\frac{\gamma \cos \theta_{e}}{h\left(x_{m}\right)} \text {. }
$$

To find a scaling relationship for the critical beam length $\ell_{c}$ at which interactions between the two beams (i.e. touching and sticking) should occur, we consider the limits of a pure line force, $\theta_{e}=\pi / 2$, and a pure curvature pressure (no line force), $\theta_{e}=0$, separately. For the case $\theta_{e}=\pi / 2,(2.1)$ may be integrated once (to eliminate the $\delta$-function) and yields $B w / \ell_{c}^{3} \sim \gamma$ so that

$$
\ell_{c, \pi / 2} \sim w^{1 / 3} \ell_{e c}^{2 / 3}
$$

where $\ell_{e c}=(B / \gamma)^{1 / 2}$ is the elastocapillary length (Bico et al. 2004) and measures the competition between minimizing bending and surface energies. In the case $\theta_{e}=0$ the analogous scaling argument applied directly to (2.1) gives $B w / \ell_{c}^{4} \sim \gamma / w$ and so

$$
\ell_{c, 0} \sim w^{1 / 2} \ell_{e c}^{1 / 2}
$$

We note that the scaling laws in (2.4) and (2.5), whilst being qualitatively similar, are quantitatively different; while the scaling law (2.5) for $\theta_{e}=0$ has frequently been given in the literature (see, for example, Bico et al. 2004), we are unaware of other occurrences of the scaling (2.4) for $\theta_{e}=\pi / 2$ having been given. However, we note that our use of beam theory requires that the slope of the beam at the critical length for touching $w / \ell_{c} \ll 1$ so that $w \ll \ell_{e c}$ and $\ell_{c, 0} \ll \ell_{c, \pi / 2}$. We thus expect that for intermediate values of $\theta_{e}$ it should be the scaling (2.5) that is the most pertinent.

\subsection{A dynamic model}

We shall see that the scaling argument given in $\S 2.1$ is informative in understanding the different static configurations of beams. However, more generally we are interested in investigating the motion of the beams in non-equilibrium situations and so require a model that couples the motion of the beams to the motion of the liquid within the channel. We neglect the inertia of the beams because of the microscopic scale of most 
practical applications. Similarly, and because of the small width-to-length ratio of many channels used in practice, we develop a dynamic model based on lubrication theory within the thin channel gap. Following the standard approximation of the equations of motion in this limit (see Leal 2007, for example), the pressure $p$ and liquid velocity $(u, v)$ satisfy

$$
\frac{\partial p}{\partial x}=\mu \frac{\partial^{2} u}{\partial y^{2}}, \quad \frac{\partial p}{\partial y}=0, \quad \frac{\partial u}{\partial x}+\frac{\partial v}{\partial y}=0 .
$$

Using no-slip boundary conditions at $y= \pm h$ we may solve (2.6 a,b) for the velocity $u(x, y, t)$ which may then be integrated from $y=0$ to $y=h(x, t)$ to obtain the half-flux

$$
Q=-\frac{h^{3}}{3 \mu} \frac{\partial p}{\partial x}
$$

Since the inertia of the beam is negligible, the pressure $p(x, t)$ is given by the rearranged version of (2.1). Finally, conservation of mass gives an evolution equation for the shape of the beam, $h(x, t)$,

$$
\frac{\partial h}{\partial t}=\frac{B}{3 \mu} \frac{\partial}{\partial x}\left(h^{3} \frac{\partial^{5} h}{\partial x^{5}}\right)
$$

valid for $0<x<x_{m}$.

Equation (2.8) is to be solved subject to clamped boundary conditions at $x=0$, i.e.

$$
h(0, t)=\frac{w}{2},\left.\quad \frac{\partial h}{\partial x}\right|_{x=0}=0
$$

as well as the requirement that there be no liquid flux through the rigid boundary at $x=0$, which, using (2.7) with (2.1) gives

$$
\left.\frac{\partial^{5} h}{\partial x^{5}}\right|_{x=0}=0
$$

At the meniscus $x=x_{m}$ the pressure within the liquid is given by the curvature of the meniscus and so we have

$$
\left.\frac{\partial^{4} h}{\partial x^{4}}\right|_{x=x_{m}}=-\frac{\gamma \cos \theta_{e}}{B} \frac{1}{h\left(x_{m}\right)} .
$$

Continuity for the shape between the wet and dry portions of the beam requires

$$
[h]_{-}^{+}=\left[\frac{\partial h}{\partial x}\right]_{-}^{+}=\left[\frac{\partial^{2} h}{\partial x^{2}}\right]_{-}^{+}=0, \quad\left[\frac{\partial^{3} h}{\partial x^{3}}\right]_{-}^{+}=-\frac{\gamma \sin \theta_{e}}{B},
$$

where $[f]_{-}^{+} \equiv f\left(x_{m}^{+}, t\right)-f\left(x_{m}^{-}, t\right)$.

The shape of the dry portion of the beam satisfies

$$
B \frac{\partial^{4} h}{\partial x^{4}}=0,
$$

with boundary conditions at the end of the beam, $x=\ell$, depending on which of the configurations shown in figure 1 the beams adopt. When the ends are separated, they are 'free' with zero bending moment and shear (Landau \& Lifschitz 1959) so that

$$
\frac{\partial^{2} h}{\partial x^{2}}=\frac{\partial^{3} h}{\partial x^{3}}=0, \quad x=\ell .
$$

However, for some parameter values we expect that the ends of the beams will touch, in 
which case 'hinged' boundary conditions are appropriate, i.e.

$$
h(\ell, t)=\left.\frac{\partial^{2} h}{\partial x^{2}}\right|_{x=\ell}=0 .
$$

For still other parameter values we expect that the ends might stick along some portion of their length $x_{c}<x<\ell$ (see figure 1c) and so

$$
h\left(x_{c}, t\right)=\left.\frac{\partial h}{\partial x}\right|_{x=x_{c}}=\left.\frac{\partial^{2} h}{\partial x^{2}}\right|_{x=x_{c}}=0
$$

for some $x_{c}$ satisfying $x_{m}<x_{c}<\ell$. Note that the continuity of $\partial^{2} h / \partial x^{2}$ at $x=x_{c}$ ensures that there is no adhesive force between the two beams (Majidi 2007).

The problem is then fully specified apart from the position of the contact line $x_{m}$. This may be found either from the local conservation of mass at the contact line or by the requirement that the total volume of liquid is conserved. We find that

$$
\frac{\mathrm{d} x_{m}}{\mathrm{~d} t}=\frac{Q}{h\left(x_{m}, t\right)}=-\left.\frac{B}{\mu} \frac{h\left(x_{m}, t\right)^{2}}{3} \frac{\partial^{5} h}{\partial x^{5}}\right|_{x_{m}} .
$$

For some parameter values we might expect that the meniscus reaches the end of the beam. As noted in the Introduction, we do not allow this possibility here and so terminate the simulations if the meniscus reaches the end of the beam.

Finally, we note that our governing equation and boundary conditions are equivalent to those studied in Aristoff et al. (2011) except for boundary condition (2.10), which ensures that the amount of fluid between the two beams remains constant. Aristoff et al. (2011) were studying a problem in which the end $x=0$ is open to a bath of liquid so that a zero pressure condition at the clamped end $x=0$ was appropriate, rather than our no flux condition (2.10).

\subsection{Non-dimensionalization}

We non-dimensionalize all length variables with the elastocapillary length $\ell_{e c}=(B / \gamma)^{1 / 2}$ and time with the elastocapillary time $t_{e c} \equiv \mu \ell_{e c} / \gamma$. In particular, we let

$$
H(X, T)=h(x, t) / \ell_{e c}, \quad(L, W, X)=(\ell, w, x) / \ell_{e c}, \quad T=t / t_{e c}, \quad P=\gamma / \ell_{e c} p .
$$

With this choice of dimensionless variables, (2.8) becomes

$$
\frac{\partial H}{\partial T}=\frac{1}{3} \frac{\partial}{\partial X}\left(H^{3} \frac{\partial^{5} H}{\partial X^{5}}\right),
$$

subject to boundary conditions

$$
H(0, T)=\frac{W}{2}, \quad H_{X}(0, T)=H_{X X X X X}(0, T)=0,
$$

and

$$
[H]_{-}^{+}=\left[H_{X}\right]_{-}^{+}=\left[H_{X X}\right]_{-}^{+}=0, \quad\left[H_{X X X}\right]_{-}^{+}=-\sin \theta_{e}, \quad H_{X X X X}\left(X_{m}, T\right)=-\frac{\cos \theta_{e}}{H\left(X_{m}\right)},
$$

where $[F]_{-}^{+} \equiv F\left(X_{m}^{+}, T\right)-F\left(X_{m}^{-}, T\right)$, as well as the appropriate conditions at the dry end of the beam, namely

$$
\begin{array}{rlrl}
H_{X X}(L, T)=H_{X X X}(L, T) & =0, & & \text { (separated ends) } \\
H(L, T)=H_{X X}(L, T)=0, & & \text { (touching ends) } \\
H\left(X_{c}, T\right)=H_{X}\left(X_{c}, T\right)=H_{X X}\left(X_{c}, T\right)=0, & & \text { (sticking ends). }
\end{array}
$$


The position of the contact line $X_{m}(T)$ evolves according to the dimensionless version of (2.16), which reads

$$
\frac{\mathrm{d} X_{m}}{\mathrm{~d} T}=-\left.\frac{H\left(X_{m}, T\right)^{2}}{3} \frac{\partial^{5} H}{\partial X^{5}}\right|_{X_{m}} .
$$

We emphasize that in this paper we assume for simplicity that the contact angle retains its equilibrium value $\theta_{e}$ and is not related to the speed at which the contact line moves.

Finally, we must apply an initial condition $H(X, 0)=H_{0}(X)$ and find an initial meniscus position $X_{m}(0)$ that satisfies the constraint on the volume of liquid trapped between the beams, i.e.

$$
V=2 \int_{0}^{X_{m}(0)} H_{0}(X) \mathrm{d} X .
$$

The conservation of mass used in the derivation of (2.18) ensures that this volume is conserved at all times and hence is consistent with the local conservation of mass argument used at $X=X_{m}$. However, we note that in doing so we are neglecting the volume of liquid that is displaced by the meniscus itself. Given that the ratio of this volume to the total volume of liquid scales like $w / \ell$, the neglect of this volume is entirely consistent with the small-aspect-ratio approximation used to justify both the use of linear beam theory and lubrication theory in the derivation of the governing equations.

The evolution equation (2.18) is to be solved on the evolving interval $0 \leqslant X \leqslant X_{m}(T)$. To facilitate the numerical solution of this equation we let $Z=X / X_{m}(T) \in[0,1]$ (this is modified to $Z=X / X_{c}(T)$ when the ends of the beams stick, as in figure 1c) transforming (2.18) to

$$
\frac{\partial H}{\partial T}=\frac{\dot{X_{m}} Z}{X_{m}} \frac{\partial H}{\partial Z}+\frac{1}{3} \frac{\partial}{\partial Z}\left[H^{3}\left(\frac{\partial^{5} H}{\partial Z^{5}}\right)\right],
$$

where $(\dot{)}$ denotes $\mathrm{d}() / \mathrm{d} T$. Note that the transformation to the rescaled interval $0 \leqslant Z \leqslant 1$ introduces an additional advective term in (2.24) that does not appear in the presentation of Aristoff et al. (2011). Equation (2.24) is then solved on a fixed mesh using the method of lines; further details of the numerical scheme employed may be found in Appendix A. The volume of liquid is monitored to give an indication of the numerical errors introduced; it is found to remain within $0.01 \%$ of its initial value for all simulations reported here.

\subsection{Equations for equilibrium}

The special case of equilibrium may be recovered from the steady case of the dynamic problem given by $(2.18)$ or by returning to the original beam equation. We have that the equilibrium beam shape $Y=H_{e}(X)$ satisfies

$$
\frac{\mathrm{d}^{4} H_{e}}{\mathrm{~d} X^{4}}=P(X)-\sin \theta_{e} \delta\left(X-X_{m}\right)
$$

where

$$
P(X)=\left\{\begin{array}{ll}
-\frac{\cos \theta_{e}}{H_{e}\left(X_{m}\right)}, & 0 \leqslant X<X_{m} \\
0, & X_{m} \leqslant X \leqslant L
\end{array} .\right.
$$

We note the pressure within the liquid $P(X)$ is negative (a capillary suction) provided that $\theta_{e}<\pi / 2$ but that it becomes positive (and hence a capillary repulsion) when $\theta_{e}>\pi / 2$.

To determine the equilibrium shape of the beam, we solve (2.25)-(2.26) subject to the boundary conditions $(2.19)-(2.20)$ (with $H(X, T)$ replaced by $H_{e}(X)$ ) at $X=0, X_{m}$, 
and the appropriate conditions from $(2.21 a)-(2.21 c)$ at $X=L$. The volume of liquid for a given equilibrium shape can be found from (2.23).

\section{A pure line force: $\theta_{e}=\pi / 2$}

In this section we isolate the effect of the surface tension-induced line force (compared to the curvature force) by setting $\theta_{e}=\pi / 2$. We shall assume that the position of the meniscus $X_{m}<L$ is given, calculate the corresponding equilibrium shape $H_{e}\left(X ; X_{m}\right)$ and then calculate the volume $V\left(X_{m}\right)$ required to produce this meniscus position using (2.23). This approach facilitates the calculation, though in reality we expect that it is the volume of liquid $V$ that is given and hence that the meniscus will move until it is at the equilibrium position for that volume (subject to the constraint that the meniscus location does not reach the end of the beam, i.e. $X_{m} / L<1$ ).

\subsection{Equilibrium configurations}

We begin by considering each of the three different possibilities illustrated in figure 1 in turn before then combining these results into a regime diagram illustrating when each of these possibilities is realized. However, recalling the scaling law (2.4) leads us to expect that the typical horizontal length scale for $\theta_{e}=\pi / 2$ is $L_{c} \sim W^{1 / 3}$. A consequence of this is that the three-dimensional parameter space $(W, V, L)$ can be reduced to a twodimensional parameter space by letting $\omega=W / L^{3}$ and $\nu=V / L^{4}$. We therefore introduce the rescaled variables $\eta=H / L^{3}, \eta_{e}=H_{e} / L^{3}, \xi=X / L, \xi_{m}=X_{m} / L$ and make use of this rescaling in what follows.

\subsubsection{Regime I: Separated ends}

Solving (2.25)-(2.26) with boundary conditions $(2.21 a)$ we find that the rescaled equilibrium beam shape is given by

$$
\eta_{e}\left(\xi ; \xi_{m}\right)=\left\{\begin{array}{lc}
\frac{\omega}{2}+\frac{\xi^{2}}{6}\left(\xi-3 \xi_{m}\right), & 0<\xi<\xi_{m} \\
\frac{\omega}{2}+\frac{\xi_{m}^{2}}{6}\left(\xi_{m}-3 \xi\right), & \xi_{m}<\xi<1
\end{array}\right.
$$

and that the corresponding (rescaled) volume of liquid is

$$
\nu\left(\xi_{m} ; \omega\right)=\omega \xi_{m}-\xi_{m}^{4} / 4
$$

For this solution to be valid we require that the beam ends do not touch, i.e. that $\eta_{e}(1)>0$, and so we must have

$$
\xi_{m}^{2}\left(3-\xi_{m}\right)<3 \omega
$$

We note that the non-monotonic form of $\nu\left(\xi_{m} ; \omega\right)$ in $(3.2)$ suggests that for some values of the volume of liquid $\nu$ there may be two valid meniscus positions $\xi_{m}$ - a simple example of the multiple equilibria that are the focus of this paper.

\subsubsection{Regime II: Touching ends}

Solving (2.25)-(2.26) with boundary conditions (2.21b) we find that the beam shape is given by

$$
\eta_{e}\left(\xi ; \xi_{m}\right)=\left\{\begin{array}{lc}
\frac{\omega}{2}+A \xi^{2}+B \xi^{3}, & 0<\xi<\xi_{m} \\
C(1-\xi)+D(1-\xi)^{3}, & \xi_{m}<\xi<1
\end{array}\right.
$$


with

$$
\begin{aligned}
& A=3 D-\frac{1}{2} \xi_{m}, \quad B=\frac{1}{6}-D \\
& C=\frac{1}{2} \xi_{m}^{2}-3 D, \quad D=-\frac{\omega}{4}+\frac{3-\xi_{m}}{12} \xi_{m}^{2} .
\end{aligned}
$$

The corresponding liquid volume is given by

$$
\nu\left(\xi_{m} ; \omega\right)=\omega \xi_{m}+\frac{2}{3} A \xi_{m}^{3}+\frac{1}{2} B \xi_{m}^{4} .
$$

For the solutions in this regime to be physically relevant, we must have that the slope of the beam at $\xi=1$ be negative, i.e. $C>0$. Furthermore, we must also ensure that the reaction force exerted by the beams on one another is in the negative sense (i.e. is a repulsive, rather than attractive, force). Hence we also require that $D>0$. To satisfy both of these constraints, we require that

$$
\xi_{m}^{2}\left(1-\xi_{m}\right)<3 \omega<\xi_{m}^{2}\left(3-\xi_{m}\right) .
$$

\subsubsection{Regime III: Sticking ends}

In the event that the ends of the beams 'stick', the first contact occurs at some unknown position $\xi=\xi_{c}=X_{c} / L$. Solving (2.25)-(2.26) with boundary conditions $(2.21 c)$ we find that the beam shape is given by

$$
\eta_{e}\left(\xi ; \xi_{m}\right)=\left\{\begin{array}{l}
\frac{\omega}{2}+A \xi^{2}+B \xi^{3}, \quad 0<\xi<\xi_{m} \\
C\left(\xi_{c}-\xi\right)^{3}, \quad \xi_{m}<\xi<\xi_{c}
\end{array}\right.
$$

where

$$
A=\frac{1}{2} \xi_{m}\left(\frac{\xi_{m}}{\xi_{c}}-1\right), \quad B=\frac{1}{6}\left(1-\frac{\xi_{m}^{2}}{\xi_{c}^{2}}\right), \quad C=\frac{1}{6} \frac{\xi_{m}^{2}}{\xi_{c}^{2}}
$$

and

$$
\xi_{c}=\xi_{m}+\frac{3 \omega}{\xi_{m}^{2}}
$$

The corresponding liquid volume is given by

$$
\nu\left(\xi_{m} ; \omega\right)=\omega \xi_{m}+\frac{2}{3} A \xi_{m}^{3}+\frac{1}{2} B \xi_{m}^{4} .
$$

For this solution to be physically realistic we need to ensure that contact occurs within the length of the beam, i.e. that $\xi_{c}<1$, which leads to the constraint

$$
3 \omega<\xi_{m}^{2}\left(1-\xi_{m}\right)
$$

We note that the reaction force in this configuration is always repulsive (as desired) since $C>0$ in (3.9). In addition, the contact point always lies beyond the meniscus position since $X_{c}>X_{m}$ from (3.10).

\subsubsection{The regime diagram}

We now bring together the above results to present a regime diagram showing the regions of parameter space in which each of the different beam configurations are possible. Because of the nature of the inequalities (3.3), (3.7) and (3.12) it is a very simple matter to illustrate the regions of $\left(\xi_{m}, \omega\right)$ space for which each regime is realized. Such a plot is shown in figure 2 .

However, such a plot has limited utility since in any practical application it is $\omega$ and $\nu$ (not $\xi_{m}$ ) that are given; it is thus the $(\omega, \nu)$ parameter space that we wish to characterize in a regime diagram. To do this, we begin by noting that the right hand side of the inequality in (3.12) has maximum value $4 / 27$ and hence that regime III is only possible 


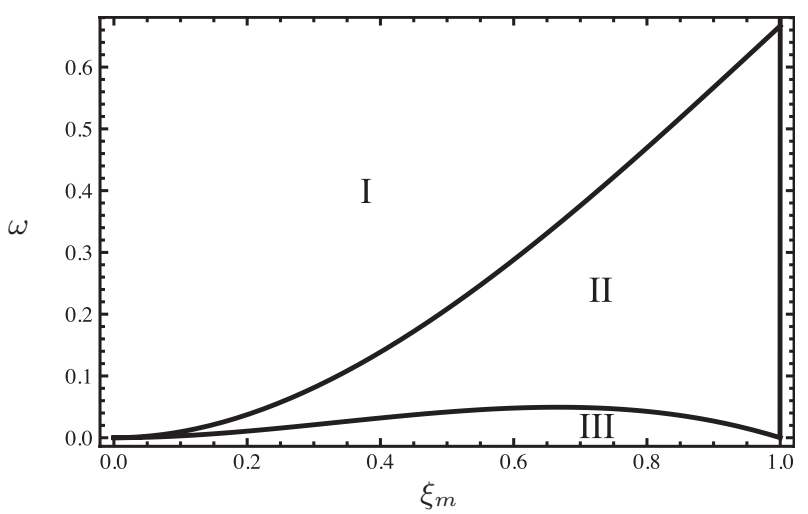

FiguRE 2. Regime diagram showing the regions of $\left(\xi_{m}, \omega\right)$ parameter space for which each of the various regimes discussed are observed. The regimes are numbered as follows: I (separated ends), II (touching ends) and III (sticking ends).

for $\omega<4 / 81$. Similarly, the right hand side of the inequality in (3.7) has a maximum value of 2 for $0 \leqslant \xi_{m} \leqslant 1$ and so regime II is only possible for $\omega<2 / 3$. Since avoiding the possibility of touching (and sticking) ends is an important practical consideration a simple design criterion would be to ensure that $\omega>2 / 3$ : within the limitations of the current model such a criterion ensures that no matter what volume of liquid is deposited the ends remain separated.

Now, the maximum liquid volume that can be accommodated in regime I is $\nu=3 \omega^{4 / 3} / 4$, which is obtained with $\xi_{m}=\omega^{1 / 3}$. This maximum is, however, not attainable for all values of $\omega$; when $\omega>1$ the optimal $\xi_{m}>1$ and hence is not physically realizable. Hence the actual maximum volume is $\nu(1 ; \omega)=\omega-1 / 4$. Similarly, for $\omega<3^{3} / 4^{3} \approx 0.422, \xi_{m}=\omega^{1 / 3}$ does not satisfy the inequality (3.3). We therefore have that regime I is possible for

$$
\nu< \begin{cases}\nu_{1}(\omega), & 0<\omega<3^{3} / 4^{3} \\ \frac{3}{4} \omega^{4 / 3}, & 3^{3} / 4^{3}<\omega<1 \\ \omega-1 / 4, & \omega>1\end{cases}
$$

where

and $\xi_{1}$ is the solution of

$$
\nu_{1}(\omega)=\xi_{1}^{3}\left(1-\frac{7}{12} \xi_{1}\right)
$$

$$
3 \omega=\xi_{1}^{2}\left(3-\xi_{1}\right) .
$$

In regime II the maximum value of $\nu$ is attained at $\xi_{m}=1$ with $\nu(1 ; \omega)=5 \omega / 8$ and hence, in regime II only, we have $\nu<5 \omega / 8$. Consideration of the inequalities (3.7) and (3.12) shows that the system may be in regime II when

$$
\begin{aligned}
\nu_{1}(\omega)<\nu<\nu_{2}(\omega) \text { or } \nu_{3}(\omega) & <\nu<5 \omega / 8, & & 0<\omega<4 / 81 \\
\nu_{1}(\omega) & <\nu<5 \omega / 8, & & 4 / 81<\omega<2 / 3
\end{aligned}
$$

where $\nu_{2}$ and $\nu_{3}$ are given by

$$
\nu_{i}(\omega)=-\frac{1}{12} \xi_{i}^{3}\left(\xi_{i}-1\right)\left[4+\xi_{i}\left(\xi_{i}-3\right)\right], \quad i=2,3,
$$

and $\xi_{2,3}$ are the two roots of

$$
3 \omega=\xi_{i}^{2}\left(1-\xi_{i}\right)
$$



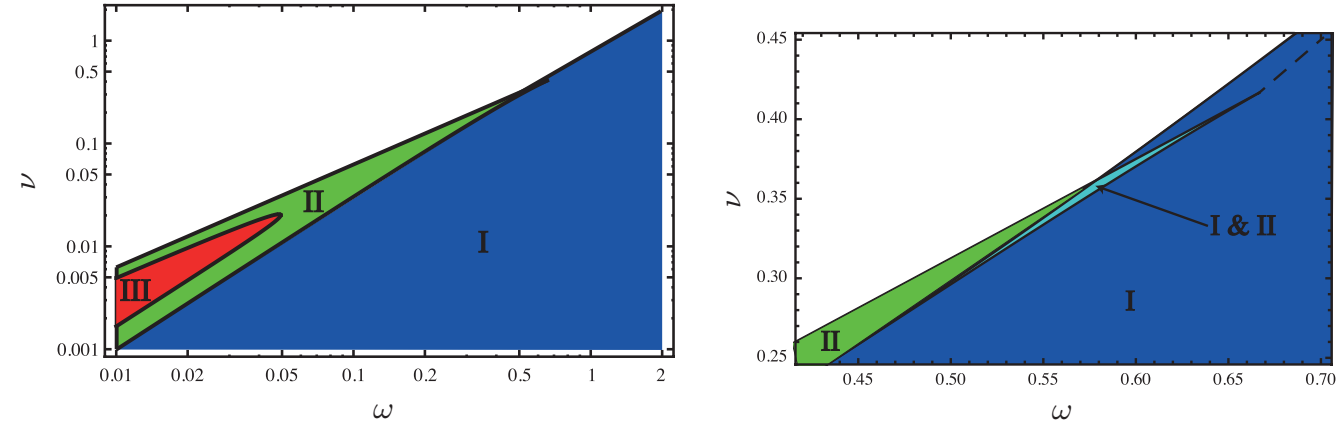

FiguRE 3. Regime diagram for $\theta_{e}=\pi / 2$ showing the regions of $(\omega, \nu)$ parameter space for which each of the various possibilities discussed are observed. A zoom in of the co-existence between states I and II is shown on the right. Above the dashed line is the region for which there are two regime I equilibria.

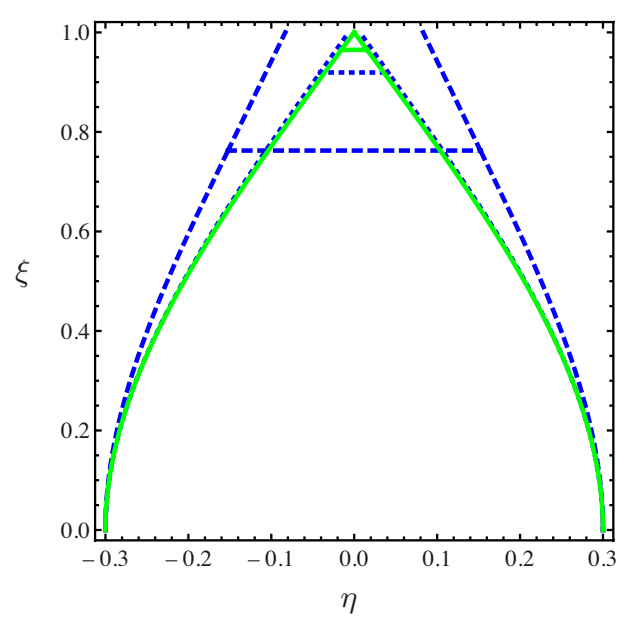

FIGURE 4. An example of the multiple equilibria that co-exist with $\theta_{e}=\pi / 2$. Here $\nu=0.373$, $\omega=0.6$. An equilibrium configuration in regime II with $\xi_{m} \approx 0.965$ (solid curve) coexists with two equilibria in regime I, which have $\xi_{m} \approx 0.763$ (dashed) and $\xi_{m} \approx 0.919$ (dotted). Note that the curves show the shapes of the deflected beams while the horizontal lines show the shape and location of the meniscus.

satisfying $0<\xi_{i}<1$. We then find that the system may be in regime III provided

$$
\nu_{2}(\omega)<\nu<\nu_{3}(\omega), \quad 0<\omega<4 / 81
$$

These results are summarized in the regime diagram shown in figure $3 \mathrm{a}$. We note that there is a very small region of $(\omega, \nu)$ space for which regimes I and II are both possible. This region is shown in more detail in figure $3 \mathrm{~b}$. Furthermore, in this region two different regime I equilibrium states are possible. To emphasize this point, figure 4 shows the three different beam shapes that are possible for $(0.6,0.373)$, which lies within this regime. We shall discuss subsequently which of the three different beam shapes the system in fact chooses. Finally, we note that there is also a small region of $(\omega, \nu)$ space for which there are two regime I equilibrium states but none in regime II or III. This is also shown in figure $3 \mathrm{~b}$ as the region above the dashed line. 
(a)

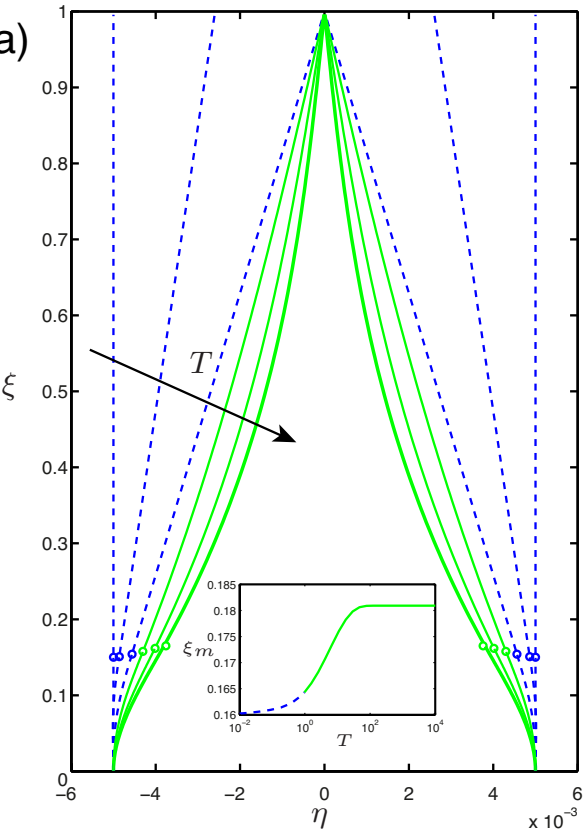

(b)

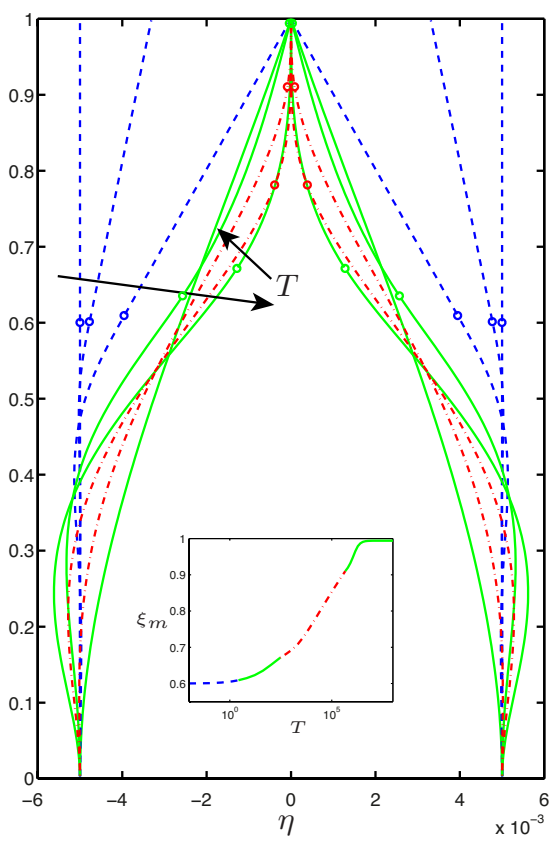

FIgURE 5. Evolution towards equilibrium in two scenarios with a single equilibrium shape. In both cases the beams are initially vertical $\eta_{0}(\xi)=\omega / 2$ and $\omega=0.01$. In (a) $\nu=0.0016$ and the beam evolves towards its steady state equilibrium with touching ends (regime II) in a straightforward manner. In (b) $\nu=0.006$ and the beam evolves through a variety of qualitatively different beam shapes before ultimately reaching its equilibrium with touching ends (regime II). The position of the meniscus $\xi=\xi_{m}$ is indicated by circles in the main figures while plots of the evolution of meniscus position, $\xi_{m}(T)$, are shown in each insets using logarithmic time scales. In each plot the regime is indicated by the use of different line styles, as follows: regime I (dashed), regime II (solid) and regime III (dot-dashed). The direction of increasing time is shown by arrows.

\subsection{Dynamic results}

To understand the stability of the multiple equilibria shown in figure 3 , as well as which equilibrium state results from a given initial condition, we use the dynamic model developed in $\S 2.3$. Typically, we take $\eta_{0}(\xi)=\omega / 2$ and $\xi_{m}(0)=\nu / \omega$ as the initial condition in our numerical scheme (described in more detail in the Appendix). The boundary conditions at the ends of the beam are those appropriate to the last known configuration of the beam and the transition between configurations, e.g. from separated ends to touching ends, is captured using event location within MATLAB. A simple example of the evolution of a system that undergoes this transition is shown in figure 5a. In this case, the beam shape evolves and the contact line moves monotonically towards the final equilibrium predicted by the regime diagram.

A more interesting example of the dynamics is demonstrated by a point within the 'upper' branch of regime II (touching ends) shown in figure 3 (i.e. $0<\omega<4 / 81$ and $\nu_{3}<\nu<5 \omega / 8$ ). In this case we observe that the beams begin with separate ends (regime I), touch (entering regime II) then stick together (regime III) before finally returning to regime II and the expected equilibrium configuration. This sequence of events is shown in figure $5 \mathrm{~b}$. If the volume is increased beyond $5 \omega / 8$ (keeping $\omega$ fixed) the beams go through the same configuration changes before the meniscus finally reaches the end of the beam, at which point our simulation stops, as discussed in $\S 2.2$. 
We now turn our attention to the behaviour in the regions of $(\omega, \nu)$ parameter space for which multiple equilibria exist. As an example of this, we take $\omega=0.6, \nu=0.373$. The three different equilibrium states possible in this case have already been plotted in figure 4 . We first investigate the stability of each of these equilibria numerically by adding a small sinusoidal perturbation to the equilibrium shape and setting this as the initial condition. We find that the regime II (touching ends) equilibrium is stable, as is the regime I (separated ends) equilibrium with the lower value of $\xi_{m}$. However, the second regime I equilibrium is unstable: the perturbed solution goes to one of the other two equilibria depending on the sign of the derivative at $\xi_{m}$. An example of this is given in figure $6 \mathrm{a}$.

It is interesting to compare this behaviour with what might have been expected on the basis of the total energy of the system. The total energy (bending plus surface energy) is given by

$$
E=\int_{0}^{1} \frac{1}{2} \eta_{\xi \xi}^{2} \mathrm{~d} \xi+\eta_{m},
$$

where $\eta_{m}=\eta\left(\xi_{m}\right)$, noting that since $\theta_{e}=\pi / 2$ we need only include the surface energy from the meniscus itself, as there is no change in the solid surface energy as the meniscus moves. We plot the energy against the volume $\nu$ for constant $\omega=0.6$ in figure $6 \mathrm{~b}$, from which we see that for volumes that permit more than one equilibrium, their energy decreases with increasing $\xi_{m}$. Taking the case considered above $(\nu=0.373)$, this means that the regime II solution is the most energetically favourable, while the regime I solution with lowest $\xi_{m}$, which numerical solutions indicate is stable, is in fact the least energetically favourable. We note that this complicates the simple picture presented by Mastrangelo \& Hsu (1993a). We have not been able to understand this result by considering the static energy landscape with a single control parameter, as presented by Mastrangelo \& Hsu (1993a). To examine this situation more carefully, therefore, we perform a linear stability analysis in the next section.

\subsection{Linear stability analysis}

We consider small perturbations of the beams from their equilibrium deflection $\eta_{e}(\xi)$ and equilibrium meniscus position $\xi_{m}^{(0)}$ by letting

$$
\eta(\xi, T)=\eta_{e}(\xi)+\varepsilon \mathrm{e}^{\sigma T} f(\xi), \quad \xi_{m}(T)=\xi_{m}^{(0)}+\varepsilon \mathrm{e}^{\sigma T} \xi_{m}^{(1)},
$$

where $\varepsilon \ll 1$. At first order in $\varepsilon$ we find that the rescaled perturbation $g(\xi)=f(\xi) / \xi_{m}^{(1)}$ satisfies

subject to

$$
\sigma g=\frac{1}{3}\left(\eta_{e}^{3} g^{\prime \prime \prime \prime \prime \prime}\right)^{\prime}
$$

$$
\begin{aligned}
g=g^{\prime}=g^{\prime \prime \prime \prime \prime} & =0 \quad \text { at } \xi=0, \\
g^{\prime \prime}+1=g^{\prime \prime \prime}=g^{\prime \prime \prime \prime} & =0 \quad \text { at } \xi=\xi_{m}^{(0)}, \\
\int_{0}^{\xi_{m}^{(0)}} g(\xi) \mathrm{d} \xi+\eta_{e}\left(\xi_{m}^{(0)}\right) & =0,
\end{aligned}
$$

for the case of separated ends, while (3.23b) must be modified accordingly for touching or sticking ends. We note that the form of the boundary conditions in $(3.23 \mathrm{~b})$ results from the Taylor expansion of the boundary conditions about the unperturbed meniscus position. 

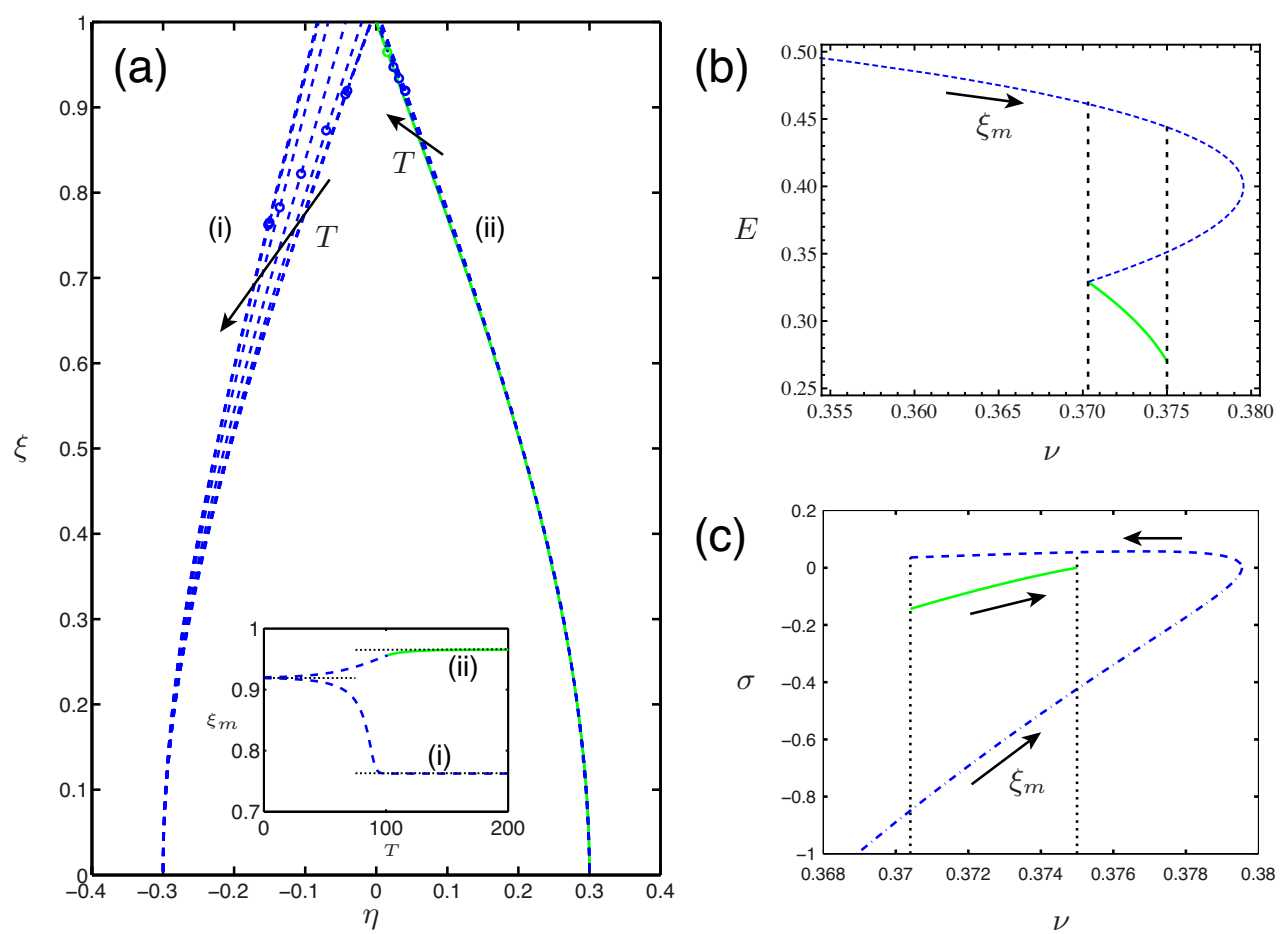

(c)

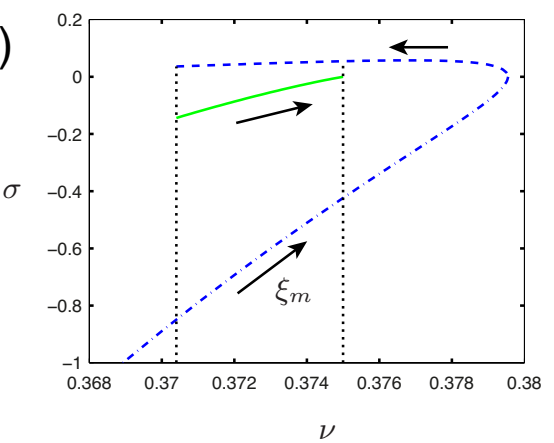

FIGURE 6. (a) Dependence on initial conditions in a scenario with multiple equilibria. Here $\nu=0.373, \omega=0.6$ so that an equilibrium in regime II (touching ends) coexists with two equilibria in regime I (see figure 4 for the shapes of these equilibria). Only two of these three equilibria are stable; starting with different small perturbations to the unstable equilibrium the beam shape tends to either (i) the second regime I configuration or (ii) the regime II configuration. Arrows show the direction of increasing time $T$. The corresponding behaviours of the meniscus position $\xi_{m}(T)$ in each case are shown in the inset. (b) Total energy $E$ of the equilibria as a function of reduced volume $\nu$ for $\omega=0.6$, showing that energy decreases as the meniscus position $\xi_{m}$ increases. (c) The growth rate $\sigma$ of perturbations to the equilibria as a function of $\nu$ for $\omega=0.6$ (the direction of increasing $\xi_{m}$ is shown by arrows). In all cases the curves corresponding to regime I (separated ends) are dashed, those corresponding to regime II (touching ends) are solid. The vertical dotted lines in (b) and (c) show the range of liquid volumes for which multiple equilibria exist.

For $\sigma \neq 0$ we may substitute (3.22) into (3.23c) to find

$$
\sigma=-\frac{1}{3} \eta_{e}^{2} g^{\prime \prime \prime \prime \prime \prime} \quad \text { at } \xi=\xi_{m}^{(0)}
$$

This expression of the conservation of mass is more convenient for numerical implementation that $(3.23 \mathrm{c})$ and so is used here.

We solve (3.22)-(3.23) using MATLAB's boundary value solver bvp4c which computes the eigenvalue $\sigma$ as part of the solution. We show the results for $w=0.6, \nu=0.373$ in figure $6 \mathrm{c}$. The results are in qualitative agreement with the results from the full numerical solution of our dynamic model: the regime I equilibrium with lower $\xi_{m}$ and the regime II equilibrium are both stable, while the regime I equilibrium with higher $\xi_{m}$ is unstable. Furthermore, this instability has a slow growth rate $(\sigma \approx 0.05)$, consistent with the $O(100)$ time scale over which the motion occurs, see inset of figure 6 a. 
4. A pure curvature force: $\theta_{e}=0$

\subsection{Equilibrium configurations}

In this section we isolate the effect of the curvature force compared to the line force from surface tension by setting $\theta_{e}=0$. We recall that the scaling law (2.5) leads us to expect that the typical horizontal length scale for $\theta_{e}=0$ is $L_{c} \sim W^{1 / 2}$. We therefore introduce new rescaled variables $\omega=W / L^{2}, \nu=V / L^{3}$ and $\eta_{e}=H_{e} / L^{2}$ whilst keeping $\xi=X / L$ and $\xi_{m}=X_{m} / L$ in what follows. We have therefore reduced the three dimensional $(W, V, L)$ parameter space to the two dimensional $(\omega, \nu)$ parameter space. However, in this case the opportunity for analytical arguments is much more limited for $\theta_{e}=0$ than was the case for $\theta_{e}=\pi / 2$ considered in $\S 3$. We shall have to explore parameter space numerically to draw the corresponding $(\omega, \nu)$ regime diagram, though it is possible to determine analytically the inequalities that must be satisfied in each region.

\subsubsection{Regime I: Separated ends}

By solving (2.25)-(2.26) with boundary conditions (2.21a) we find that the beam shape is given by

$$
\eta_{e}\left(\xi ; \xi_{m}\right)=\left\{\begin{array}{l}
\eta_{m}+\frac{1}{24 \eta_{m}}\left[4 \xi_{m}^{3}\left(\xi_{m}-\xi\right)-\left(\xi_{m}-\xi\right)^{4}\right], \quad 0<\xi<\xi_{m} \\
\eta_{m}+\frac{\xi_{m}^{3}}{6 \eta_{m}}\left(\xi_{m}-\xi\right), \quad \xi_{m}<\xi<1
\end{array}\right.
$$

with the deformation at the meniscus, $\eta_{m}$, satisfying

$$
\eta_{m}^{2}-\frac{\omega}{2} \eta_{m}+\frac{\xi_{m}^{4}}{8}=0
$$

For this solution to be valid we must have that $\eta_{e}(1)>0$, i.e. $\xi_{m}=X_{m} / L$ must satisfy

$$
\omega \eta_{m}>\frac{\xi_{m}^{3}}{12}\left(4-\xi_{m}\right) \text {. }
$$

Finally, the (rescaled) volume is given by

$$
\nu=2 \eta_{m} \xi_{m}+\frac{3}{20} \frac{\xi_{m}^{5}}{\eta_{m}}-L \times \frac{\pi}{2} \eta_{m}^{2}
$$

In (4.4) we have included, for the time being, the volume of liquid that is displaced by the meniscus.

\subsubsection{Regime II: Touching ends}

By solving (2.25)-(2.26) with boundary conditions $(2.21 b)$ we find that the rescaled equilibrium beam shape $\eta_{e}=H_{e} / L^{2}$ is given by

$$
\eta_{e}\left(\xi ; \xi_{m}\right)=\left\{\begin{array}{l}
-\frac{\left(\xi_{m}-\xi\right)^{4}}{24 \eta_{m}}+A(1-\xi)+B(1-\xi)^{3}, \quad 0<\xi<\xi_{m} \\
A(1-\xi)+B(1-\xi)^{3}, \quad \xi_{m}<\xi<1
\end{array}\right.
$$

where

$$
A=\frac{3 \omega}{4}+\frac{\xi_{m}^{3}}{48 \eta_{m}}\left(3 \xi_{m}-4\right), \quad B=\frac{1}{3}\left(\frac{\xi_{m}^{3}}{6 \eta_{m}}-A\right),
$$

and the rescaled meniscus radius $\eta_{m}$ is the solution of the quadratic equation

$$
\eta_{m}^{2}-\frac{\omega}{2}\left(1-\xi_{m}\right)\left(1+\xi_{m}-\xi_{m}^{2} / 2\right) \eta_{m}+\frac{\xi_{m}^{4}}{48}\left(1-\xi_{m}\right)\left(6-6 \xi_{m}+\xi_{m}^{2}\right)=0 .
$$


The corresponding volume of liquid trapped is given by

$$
\nu=-\frac{\xi_{m}^{5}}{60 \eta_{m}}+A \xi_{m}\left(2-\xi_{m}\right)+\frac{1}{2} B \xi_{m}\left(4-6 \xi_{m}+4 \xi_{m}^{2}-\xi_{m}^{3}\right)-L \times \frac{\pi}{2} \eta_{m}^{2} .
$$

To have physical solutions in this regime, we must have that the slope of the beam at the end be negative, i.e. $A>0$. Furthermore, we must also ensure that the reaction force is in the negative sense (i.e. is a repulsive, rather than attractive, force). Hence we also require that $B>0$. To satisfy both of these constraints, we require that

$$
\frac{1}{12}\left(\frac{4}{3}-\xi_{m}\right) \xi_{m}^{3}<\omega \eta_{m}<\frac{1}{12}\left(4-\xi_{m}\right) \xi_{m}^{3} .
$$

\subsubsection{Regime III: Sticking ends}

In the case of sticking beams, the first contact occurs at some unknown position $\xi=\xi_{c}=X_{c} / L$. Solving (2.25)-(2.26) with boundary conditions $(2.21 c)$ we find that the beam shape is given by

$$
\eta_{e}\left(\xi ; \xi_{m}\right)=\left\{\begin{array}{l}
-\frac{1}{24 \eta_{m}}\left(\xi_{m}-\xi\right)^{4}+A\left(\xi_{c}-\xi\right)^{3}, \quad 0<\xi<\xi_{m} \\
A\left(\xi_{c}-\xi\right)^{3}, \quad \xi_{m}<\xi<\xi_{c}
\end{array}\right.
$$

where

$$
A \xi_{c}^{3}=\frac{\omega}{2}+\frac{\xi_{m}^{4}}{24 \eta_{m}}, \quad \xi_{c}=\frac{3}{4} \xi_{m}+9 \frac{\omega \eta_{m}}{\xi_{m}^{3}},
$$

with $\xi_{c}$ and $\eta_{m}$ being found as the solution of the quartic

$$
18 \eta_{m}^{2}=\frac{\left(9 \omega \eta_{m}-\xi_{m}^{4} / 4\right)^{3}}{\left(3 \xi_{m}^{4} / 4+9 \omega \eta_{m}\right)^{2}}
$$

The corresponding liquid volume is given by

$$
\nu=-\frac{\xi_{m}^{5}}{60 \eta_{m}}+\frac{1}{4}\left(\omega+\frac{\xi_{m}^{4}}{12 \eta_{m}}\right) \xi_{c}\left[1-\left(\frac{\xi_{c}-\xi_{m}}{\xi_{c}}\right)^{4}\right]-L \times \frac{\pi}{2} \eta_{m}^{2} .
$$

For contact we need to ensure that $\xi_{m}<\xi_{c}<1$, which using (4.11) may easily be rearranged to give

$$
\frac{1}{36} \xi_{m}^{4}<\omega \eta_{m}<\frac{1}{12} \xi_{m}^{3}\left(\frac{4}{3}-\xi_{m}\right)
$$

\subsubsection{Regime diagram}

Here we collate the restrictions on $\nu$ and $\omega$ derived above to present a regime diagram describing when each of the three regimes I, II and III are observed. We note that at present the expressions for the rescaled liquid volume $\nu$ presented above all involve $L$ explicitly. However, since we expect that $\omega=W / L^{2}=O(1)$ at the transitions between the different regimes and we have already assumed that $W / L \ll 1$ to make use of linear beam theory and lubrication theory, we must in fact have that $L \ll 1$. It is thus selfconsistent to neglect the volume displaced by the meniscus $\sim \eta_{m}^{2} L \sim \omega^{2} L \ll 1$, and we shall do this henceforth. Finally, we note that the limit $L \ll 1$ is likely to be the limit of most practical interest, since it corresponds to relatively rigid beams; very floppy beams, $L \gg 1$, are unlikely to be of much practical utility.

Now, the inequalities (4.3), (4.9) and (4.14) involve not just $\omega$ and $\nu$ but also $\eta_{m}$, which itself is a function of both $\omega$ and $\xi_{m}$. We are therefore unable to construct simple inequalities to characterise the $(\omega, \nu)$ parameter space and so explore the possible existence 

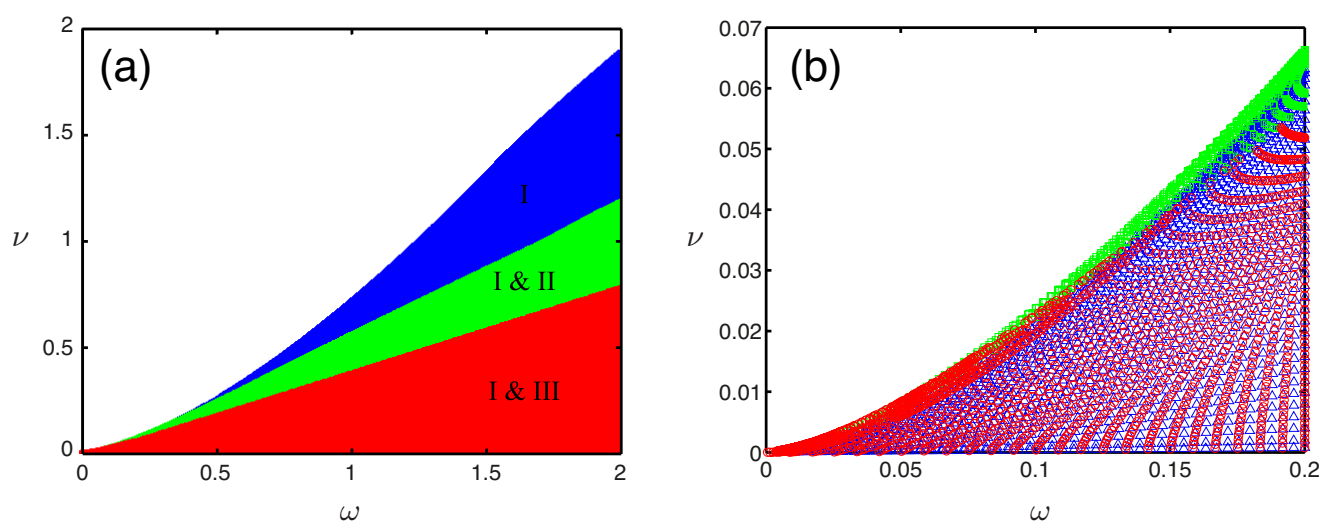

FIgURE 7. (a) Regime diagram showing the regions of $(\omega, \nu)$ parameter space for which each of the various equilibrium beam configurations are observed for $\theta_{e}=0$. (b) A zoom in of the bottom left of (a) showing that there are regions in which only regime II or regime III are possible. Points corresponding to regime I, II, and II are plotted as triangles, squares, and circles respectively.

of equilibrium states by numerical means. We show in figure 7 the regime diagram computed numerically using $\mathcal{O}\left(10^{4}\right)$ points. We again note that there appear to be regions of $(\omega, \nu)$ parameter space for which multiple regimes are possible. Indeed, provided a steady state exists it may be seen that there are always two possible configurations, possibly of the same type, while there is a small region for which four solutions exist (two regime I and two regime II). This is reminiscent of the $\theta_{e}=\pi / 2$ case examined previously, where there was a small region in $(\omega, \nu)$-space for which there are three steady states instead of one. Given that $\eta$ is in general a quartic in $\xi$, while for $\theta_{e}=\pi / 2$ it is reduced to a cubic, it is perhaps not surprising that there is generally an additional steady state for $\theta_{e}=0$ compared with $\theta_{e}=\pi / 2$.

Some examples of the multiplicity of equilibrium states are shown in figure 8 . In this case, $\omega=0.02$ and we see that by increasing the volume of liquid we pass from the coexistence of a regime I and a regime III equilibrium state, to the coexistence of a regime II and a regime III state, to two regime III states. Finally, there is too much liquid and no equilibrium states exist.

\subsection{Dynamic results}

To understand the stability of the multiple equilibrium states, we again turn to the dynamic model developed in $§ 2.3$. In general, the same qualitative behaviour is found as in the $\theta_{e}=\pi / 2$ case, with the system evolving from an arbitrary initial condition to an equilibrium state. For cases in which there are exactly two steady states, the system is observed to always evolve to the same equilibrium state - the other equilibrium is unstable. We confirm this observation based on numerical results by repeating the stability analysis of $\S 3.3$, so that the perturbation to the steady state profile satisfies the eigenvalue problem $(3.22)-(3.23 \mathrm{a}, \mathrm{c})$ while the boundary conditions $(3.23 \mathrm{~b})$ are modified to

$$
g^{\prime \prime}=\eta_{e}\left(\xi_{m}^{(0)}\right) g^{\prime \prime \prime}-1=\eta_{e}\left(\xi_{m}^{(0)}\right)^{2} g^{\prime \prime \prime \prime \prime \prime}-\eta_{e}^{\prime}\left(\xi_{m}^{(0)}\right)-g=0 \quad \text { at } \xi=\xi_{m}^{(0)},
$$

for the case of touching ends; similar expressions hold for the case of touching or sticking ends. The stability calculation reveals the growth rate $\sigma$ for each configuration as a function of volume $\nu$ for a given value of $\omega$; a typical example for $\omega=0.02$ is given 
18

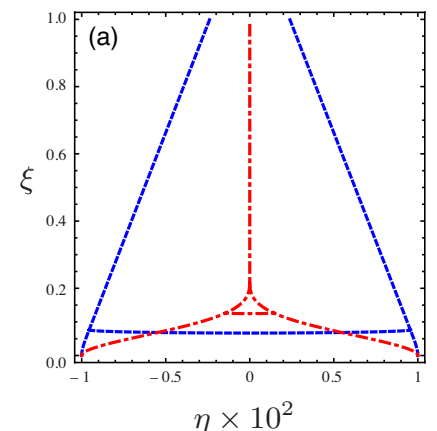

M. Taroni \&S D. Vella

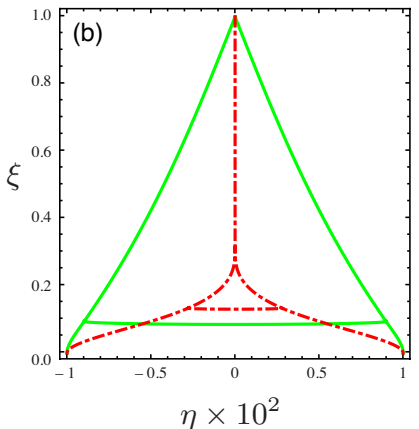

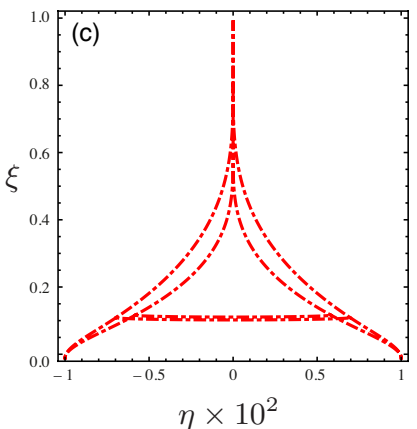

FIGURE 8. Three examples of the multiple equilibria that exist with $\theta_{e}=0$ and $\omega=0.02$. In (a) $\nu=0.0015$ : an equilibrium in regime I $\left(\xi_{m} \approx 0.076\right)$ coexists with one in regime III $\left(\xi_{m} \approx 0.126\right)$. In (b) $\nu=0.0018$ and an equilibrium in regime II $\left(\xi_{m}=0.0939\right)$ coexists with one in regime III $\left(\xi_{m} \approx 0.133\right)$ In (c) $\nu=0.0021$ and two equilibria in regime III coexist $\left(\xi_{m} \approx 0.120,0.127\right)$. In each plot the three regimes are differentiated using different line styles as follows: regime I (dashed), regime II (solid), and regime III (dot-dashed). Note that the shapes of both the menisci and the bent beams are shown. The menisci are, in fact, semicircular but appear almost horizontal because of the difference in horizontal and vertical scales.

in figure 9a. We observe that where different regimes coexist a regime I equilibrium is always more stable (more negative $\sigma$ ) than one in regime II, which is in turn more stable than an equilibrium in regime III with which it coexists. This observation is important for practical purposes since it suggests that in general the system will choose a stable equilibrium in either regime I or II while the remaining regime III configuration will be unstable. There is only a very narrow window of parameter space for which only regime III is possible; however, for such parameter values one of the regime III states is stable and the other unstable.

There is also a small region of $(\omega, \nu)$ parameter space in which there are four possible equilibrium states: two of regime I and two of regime II. In this region of parameter space the stability analysis reveals that one of each type (regime I and II) is stable, the other being unstable. This may be seen in figure 10b, where we plot the linear growth rate $\sigma$ of perturbations to the steady state as a function of $\nu$ for $\omega=0.6$.

It is again useful to consider the energy of the system. In this case, $\theta_{e}=0$, the solidliquid and solid-air surface energies are no longer the same and so the energy of the system is given by

$$
E=\int_{0}^{1} \frac{1}{2} \eta_{\xi \xi}^{2} \mathrm{~d} \xi+\frac{1}{2} \pi \eta_{m}-\xi_{m}
$$

where the extra term arises because of this difference in surface energies. Note that this energy applies equally to regime III as to regimes I and II since our model does not account for an additional attractive or repulsive force between the two beams, which would require a discontinuity in curvature at $\xi=\xi_{c}$ (Majidi 2007). The energy of the system is plotted as a function of $\nu$ in figure $9 \mathrm{~b}$ for $\omega=0.02$ and in figure 10c for $\omega=0.6$ and should be compared with the corresponding $(\sigma, \nu)$ plots. Taking as an example $\omega=0.02$, we see that for $\nu \gtrsim 0.00194$ the equilibrium with lowest energy is in fact unstable, and vice-versa.

\section{Discussion}

We have presented theoretical results demonstrating how a seemingly simple elastocapillary system (a drop placed between two flexible sheets) can exhibit a surprisingly 

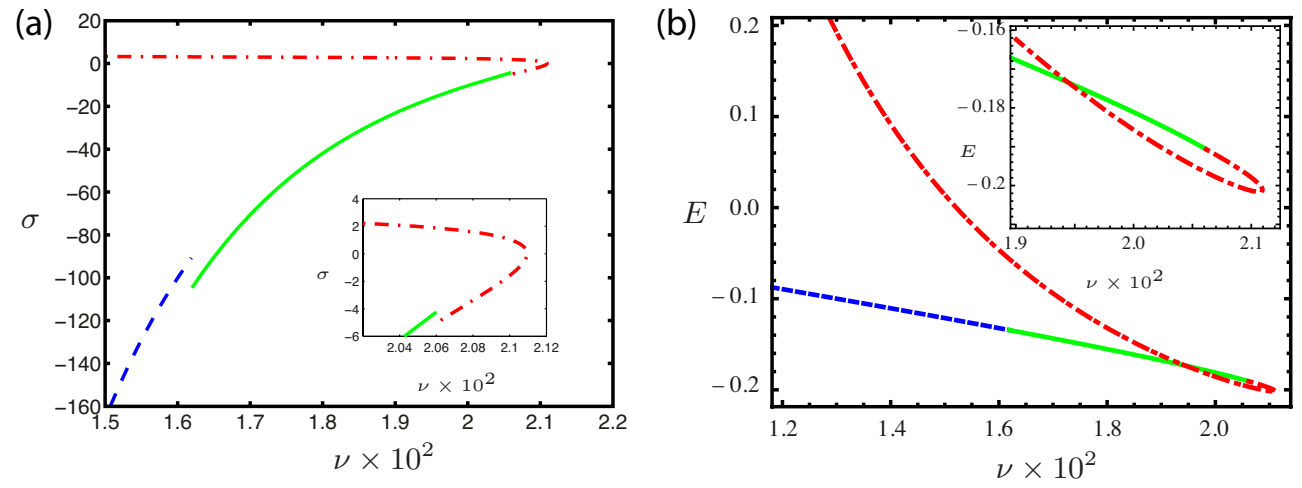

FiguRE 9. (a) Growth rate $\sigma$ of perturbations to the steady state shapes and (b) the total energy of these steady states as functions of $\nu$ for $\omega=0.02$ and $\theta_{e}=0$. In both cases the three regimes are differentiated by using different line styles - regime I (dashed), regime II (solid) and regime III (dot-dashed). Note in (a) that the growth rate $\sigma$ is discontinuous as the configuration of the beams changes since the boundary conditions to which the beam is subject also change.
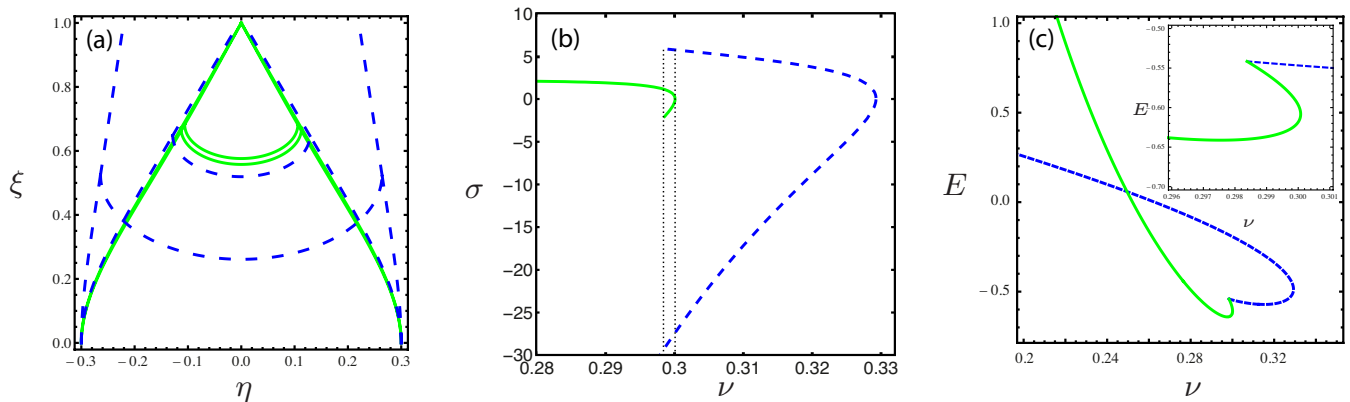

FiguRE 10. Results for $\theta_{e}=0$ and $\omega=0.6$. (a) An equilibrium scenario in which four steady states are possible with $\nu=0.3$. (b) Growth rate $\sigma$ of perturbations to the equilibrium solutions and (c) the total energy of the equilibria as functions of $\nu$. In all cases the curves corresponding to the regime I steady states are dashed, while those corresponding to the regime II steady state are solid.

rich behaviour. In particular, we have shown that in certain circumstances multiple equilibrium configurations exist and that more than one of these configurations can be stable. We have also seen that energy considerations are not necessarily enough to predict which equilibria are stable and which unstable; we have seen that for some volumes $\nu$ and widths $\omega$ there are equilibrium meniscus positions $\xi_{m}^{a}<\xi_{m}^{b}<\xi_{m}^{c}$ with energies $E\left(\xi_{m}^{a}\right)>E\left(\xi_{m}^{b}\right)>E\left(\xi_{m}^{c}\right)$ but the $\xi_{m}^{b}$ equilibrium is unstable. This appears to be in contrast to the purely static analyses used in related systems previously where it was assumed that in the absence of an explicit energy maximum the system will evolve to the global minimum of energy (Mastrangelo \& Hsu 1993a). However, the energy landscape in this case has only three disconnected points and so stability can only be studied through a dynamic calculations, which we have performed.

We have also investigated the effect of the equilibrium contact angle $\theta_{e}$ by considering the two forces on the beam generated by the interfacial tension $\gamma$ separately: for $\theta_{e}=0$ there is only a pressure force due to the curvature of the interface, while for $\theta_{e}=\pi / 2$ there is only a line force acting at the contact line position $x_{m}$. For both these limits it is possible to characterize the steady state of the system in terms of just two parameters, the reduced channel width, $\omega$, and the reduced volume of liquid, $\nu$. Although the regime 
diagrams, presented in figures 3 and 7, appear different at first glance, they are in fact quite similar once the stability of the equilibria is taken into account. In particular, we see that for sufficiently large $\omega\left(>2 / 3\right.$ for $\left.\theta_{e}=\pi / 2\right)$ we expect the system to adopt a configuration with separated ends - although touching/sticking configurations often coexist with a separated end configuration for $\theta_{e}=0$, they are always unstable. However, such configurations can become stable once $\omega$ is sufficiently small.

For intermediate values of $\theta_{e}$ the ratio of the line force to the curvature pressure force scales like $\tan \theta_{e} w / x_{m}$, and so depends on the contact angle, $\theta_{e}$, the width of the channel and the volume of liquid deposited. Nevertheless, for thin channels a simple scaling analysis suggests that in most practical situations we expect it to be the curvature pressure that dictates the critical beam length $\ell_{c}$ at which touching should occur, giving rise to the well-known scaling law $\ell_{c} \sim w^{1 / 2} \ell_{e c}^{1 / 2}$ (Bico et al. 2004).

In terms of practical applications in MEMS manufacture, our fixed-volume theory may be relevant to situations involving evaporation provided that the evaporation rate of the rinse liquid is sufficiently slow $\left(\ll 1 / t_{e c}\right)$. In this case once initial equilibrium is reached, the evolution of the beams as the volume of the drop decreases is quasi-steady. Although our results do not provide an obvious mechanism for the collapse of microchannels under slow evaporation (i.e. a switch from regime I to regime II or III under decreasing $\nu$ ), they do show that touching/sticking regimes are to be expected, at least for small $\omega$. Furthermore, our numerical results suggest that the evolution to equilibrium can be very slow, suggesting that the dynamics of evaporation may play an important role and should be included explicitly.

Despite its draw-backs, it is the simple nature of our model that has allowed us to completely characterize the interaction of elastic and capillary forces in this idealized system, and so offers an excellent starting point for theoretical investigations into more realistic scenarios. In particular, it would be possible to develop our model to take account of more realistic geometries, such as a whole series of micro-pillars, each one being pulled on either side by its neighbours (Tanaka et al. 1993), or a two-dimensional array of micro-pillars, as in the experiments of Chandra \& Yang (2009); Pokroy et al. (2009).

This publication was based on work supported in part by Award No KUK-C1-01304, made by King Abdullah University of Science and Technology (KAUST). We also thank Martin Mihelich for his contribution to the early stages of this work as a summer student supported by an Oppenheimer Early Career Fellowship (D.V.), and Kiran Singh for comments on an earlier draft.

\section{Appendix A. Numerical solution of evolution equations}

As noted in $\$ 2.3$, we solve the partial differential equation (2.18) by mapping the moving domain $X \in\left[0, X_{m}(T)\right]$ to $Z \in[0,1]$. We achieve this by letting $Z=X / X_{m}(T)$ and using the chain rule

$$
\left(\frac{\partial}{\partial T}\right)_{X}=\left(\frac{\partial}{\partial T}\right)_{Z}-\frac{\dot{X_{m} Z}}{X_{m}}\left(\frac{\partial}{\partial Z}\right)_{T}, \quad\left(\frac{\partial}{\partial X}\right)_{T}=\frac{1}{X_{m}}\left(\frac{\partial}{\partial Z}\right)_{T}
$$

where $\left(\dot{)}\right.$ denotes $\mathrm{d}() / \mathrm{d} T$ and $(\partial / \partial a)_{b}$ denotes partial differentiation with respect to $a$ whilst holding $b$ fixed. We see that this transformation of variables introduces an additional advective term to (2.18). In addition, we introduce the quantity

$$
U(Z, T)=H(Z, T) X_{m}(T)
$$




\begin{tabular}{ccc}
\hline Current regime & Event & New regime \\
\hline I & $H(L) \leqslant 0$ & II \\
II & $H(L)>0$ & I \\
II & $H_{X}\left(X_{m}\right) \geqslant 0$ & III \\
III & $X_{c}>L$ & II \\
I-III & $X_{m}>L$ & Stop \\
\hline
\end{tabular}

TABLE 1. Event functions used to change regime in dynamic model.

so that the governing equation (2.18) may be rewritten in the form

$$
\frac{\partial U}{\partial T}+\frac{\partial Q}{\partial Z}=0
$$

with $Q$ representing a horizontal flux.

We discretize the spatial domain into $N+1$ cells of equal width $\Delta Z$ where the approximate value of $U$ in the $j$-th cell, denoted $U_{j}$, is evaluated at the mid-point of the cell, while the fluxes $Q_{j \pm 1 / 2}$ are evaluated at the end-points. Spatial discretization of (A 2) using central finite differences then leads to a set of ordinary differential equations

$$
\frac{\partial U_{j}}{\partial T}=-\frac{1}{\Delta Z}\left(Q_{j+1 / 2}-Q_{j-1 / 2}\right), \quad j=1,2, \ldots N
$$

together with

$$
\frac{\mathrm{d} X_{m}}{\mathrm{~d} T}=-\frac{\left[U^{2} U_{Z Z Z Z Z}\right]_{N+1 / 2}}{3 X_{m}^{8}},
$$

while the remaining six boundary conditions are applied through the addition of three ghost points on either side of the boundary. The system of ordinary differential equations (A 3)-(A 4) is solved using MATLAB's stiff solver ode15s, with the Jacobian being calculated using complex step differentiation (using the algorithm detailed by Shampine 2007) and full advantage being taken of the sparsity of the system.

Finally, we must take care to ensure that we are using the correct boundary conditions for the ends of the beam, i.e. that the beam is in the correct regime. This is done using event functions as outlined in Table 1 (for clarity these are written in terms of the original variables), with the boundary conditions at $Z=1$ changed appropriately. In particular, we note that the computation was stopped if the meniscus reached the end of the beam.

\section{REFERENCES}

Aristoff, J. M., Duprat, C. \& Stone, H. A. 2011 Elastocapillary imbibition. Int. J. Nonlinear Mech. 46 (4), 648-656.

Berkowski, K. L., Plunkett, K. N., Yu, Q. \& Moore, J. S. 2005 Introduction to photolithography: Preparation of microscale polymer silhouettes. J. Chem. Ed. 82, 1365-1369.

Bico, J., Roman, B., Moulin, L. \& Boudaoud, A. 2004 Elastocapillary coalescence in wet hair. Nature 432, 690.

Chandra, D. \& YAng, S. 2009 Capillary-force-induced clustering of micropillar arrays: is it caused by isolated capillary bridges or by the lateral capillary meniscus interaction force? Langmuir 25 (18), 1043010434. 
Delamarche, E., Schmid, H., Michel, B. \& Biebuyck, Hans 1997 Stability of molded polydimethylsiloxane microstructures. Adv. Mater. 9, 741-746.

Duprat, C., Aristoff, J. M. \& Stone, H. A. 2011 Dynamics of elastocapillary rise. J. Fluid Mech. 679, 641-654.

Farshid Chini, S. \& Amirfazli, A. 2010 Understanding pattern collapse in photolithography process due to capillary forces. Langmuir 26 (16), 1370713714.

van Honschoten, J.W., Escalante, M., Tas, N.R., Jansen, H.V. \& Elwenspoek, M. 2007 Elastocapillary filling of deformable nanochannels. J. Appl. Phys. 101, 094310.

Kim, H.-Y. \& Mahadevan, L. 2006 Capillary rise between elastic sheets. J. Fluid Mech. 548, $141-150$.

Kwon, H.-M., Kim, H.-Y., Puell, J. \& Mahadevan, L. 2008 Equilibrium of an elastically confined liquid drop. J. Appl. Phys. 103, 093519.

Landau, L. D. \& Lifschitz, E. M. 1959 Theory of Elasticity. Pergamon Press, Oxford, UK.

Leal, L. G. 2007 Advanced Transport Phenomena. New York: Cambridge University Press.

LeE, H-J, PARK, J-T, Yoo, J, An, I \& OH, H-K 2002 Resist pattern collapse with top rounding resist profile. Jpn. J. Appl. Phys. 42, 3922-3927.

MAJIDI, C. 2007 Remarks on formulating an adhesion problem using Euler's elastica. Mech. Res. Comm. 34, 85-90.

Mansfield, E. H., Sepangi, H. R. \& Eastwood, E. A. 1997 Equilibrium and mutual attraction or repulsion of objects supported by surface tension. Phil. Trans. R. Soc. Lond. A 355, 869-919.

Mastrangelo, C. H \& Hsu, H. 1993 a Mechanical stability and adhesion of microstructures under capillary forces - part i: basic theory. J. Microelectromech. Sys. 2, 33-43.

Mastrangelo, C. H \& Hsu, H. $1993 b$ Mechanical stability and adhesion of microstructures under capillary forces-part ii: experiments. J. Microelectromech. Sys. 2, 44-55.

Pokroy, B., Kang, S. G., Mahadevan, L. \& Aizenberg, J. 2009 Self-organization of a mesoscale bristle into ordered, hierarchical helical assemblies. Science 323, 237-240.

Py, C., Reverdy, P., Doppler, L., Bico, J., Roman, B. \& Baroud, C. N. 2007 Capillary origami: Spontaneous wrapping of a droplet with an elastic sheet. Phys. Rev. Lett. 98, 156103.

Raccurt, O., Tardif, F., Arnaud D'Avitaya, F. \& Vareine, T. 2004 Influence of liquid surface tension on stiction of SOI MEMS. J. Micromech. Microeng. 14, 1083-1090.

Shampine, L. F. 2007 Accurate numerical derivatives in Matlab. ACM Trans. Math. Softw. 33, 26.

van Spengen, W. M., Puers, R. \& de Wolf, I. 2002 A physical model to predict stiction in MEMS. J. Micromech. Microeng. 12, 702-713.

Tanaka, T., Morigami, M. \& Atoda, N. 1993 Mechanism of resist pattern collapse during development process. Jpn. J. Appl. Phys. 31, 6059-6064.

Xia, Y. \& Whitesides, G. M. 1998 Soft lithography. Annu. Rev. Mater. Sci. 28, 153-184. 



\section{RECENT REPORTS}

70/11 The Capillary Interaction Between Two Vertical Cylinders Cooray

Cicuta

Vella

71/11 Nonuniqueness in a minimal model for cell motility

Gallimore

Whiteley

Waters

King

Oliver

72/11 Symmetry of uniaxial global Landau-de Gennes minimizers in the

Henao theory of nematic liquid crystals

Majumdar

73/11 Filling of a Poisson trap by a population of random intermittent searchers

Bressloff

Newby

01/12 Mechanical growth and morphogenesis of seashells

Moulton

Goriely

Chirat

02/12 How linear features alter predator movement and the functional response

McKenzie

Merrill

Spiteri

Lewis

03/12 The Fourier transform of tubular densities

Prior

Goriely

04/12 Numerical studies of homogenization under a fast cellular flow. Iyer

Zygalakis

05/12 Solute transport within porous biofilms: diffusion or dispersion? Davit

Byrne

Osborne

Pitt-Francis

Gavaghan

Quintard

65/11 Adaptive Finite Element Method Assisted by Stochastic Simulation of Chemical Systems

Cotter

Vejchodsky

Erban

06/12 Effects of intrinsic stochasticity on delayed reaction-diffusion patterning systems

Woolley

Baker

Gaffney

Maini

Seirin-Lee

07/12 Axial Dispersion via Shear-enhanced Diffusion in Colloidal Sus-

Griffiths pensions

Stone

08/12 Qualitative Analysis of an Integro-Differential Equation Model of

Jaina Periodic Chemotherapy

Byrne

09/12 Modeling Stem/Progenitor Cell-Induced Neovascularization and

Jain Oxygenation 
12/12 Modelling temperature-dependent larval development and subse-

Wheeler quent demographic Allee effects in adult populations of the alpine butterfly Parnassius smintheus

Bampfylde

Lewis

13/12 Putting "space" back into spatial ecology

Fortin

Peres-Neto

Lewis

14/12 Wildlife disease elimination and density dependence

Potapova

Merrill

Lewis

15/12 Spreading Speed, Traveling Waves, and Minimal Domain Size in Impulsive Reaction-diffusion Models

Lewis

$\mathrm{Li}$

16/12 MCMC methods for functions modifying old algorithms to make them faster

Cotter

Roberts

Stuart

White

17/12 Weyl Geometry and the Nonlinear Mechanics of Distributed Point

Yavari Defects

Goriely

18/12 A note on oblique water entry

Moore

Howison

Ockendon

Oliver

19/12 Calculus on surfaces with general closest point functions

März

Macdonald

Copies of these, and any other OCCAM reports can be obtained from:

Oxford Centre for Collaborative Applied Mathematics

Mathematical Institute

24 - 29 St Giles'

Oxford

OX1 3LB

England

www.maths.ox.ac.uk/occam 